doi: $10.2306 /$ scienceasia1513-1874.2013.39.316

\title{
Some subsemigroups of the full transformation semigroups
}

\author{
Chaiwat Namnak*, Ekkachai Laysirikul \\ Department of Mathematics, Faculty of Science, Naresuan University, Phitsanulok 65000 Thailand
}

*Corresponding author, e-mail: chaiwatn@nu.ac.th

Received 13 Sep 2012

Accepted 2 May 2013

\begin{abstract}
A nonempty subset $T$ of a semigroup $S$ is called a subsemigroup of $S$ if $T^{2} \subseteq T$, that is, $x y \in T$ for all $x, y \in T$. For a nonempty set $X$, let $T(X)$ be the semigroup of the full transformations on $X$, which consists of all functions from $X$ into $X$ with composition as the semigroup operation. The purpose of this paper is to define various subsemigroups of $T(X)$. Necessary and sufficient conditions are given for these subsemigroups of $T(X)$ to be equal.
\end{abstract}

KEYWORDS: equivalence relation, totally ordered set, transformation semigroup

\section{INTRODUCTION}

Let $S$ be a semigroup. A nonempty subset $T$ of $S$ is called a subsemigroup of $S$ if $T^{2} \subseteq T$, that is, $x y \in T$ for all $x, y \in T$.

Let $X$ be a nonempty set and let $T(X)$ denote the semigroup of the full transformations from $X$ into itself under composition of mappings.

For a totally ordered set $(X, \leqslant)$, let $E$ be an equivalence relation on $X$. Higgins ${ }^{1}$, Namnak and Laysirikul ${ }^{2}$ and $\mathrm{Pei}^{3}$ have studied the subsemigroups of $T(X)$ as follows.

$$
\begin{aligned}
& T_{\mathrm{O}}(X)=\{\alpha \in T(X): \forall x, y \in X, x \leqslant y \\
&\text { implies } x \alpha \leqslant y \alpha\} \\
& T_{\mathrm{R}}(X)=\{\alpha \in T(X): \forall x \in X, x \alpha \leqslant x\} \\
& T_{\mathrm{SE}}(X)=\{\alpha \in T(X): \forall x \in X,(x, x \alpha) \in E\}, \\
& T_{\mathrm{E}}(X)=\{\alpha \in T(X): \forall x, y \in X,(x, y) \in E \\
&\text { implies }(x \alpha, y \alpha) \in E\} .
\end{aligned}
$$

All subsemigroups clearly contain $i_{X}$ where $i_{X}$ is the identity map on $X$. Next, we define subsets of $T(X)$ by

$$
\begin{aligned}
T_{\mathrm{ER}}(X) & =T_{\mathrm{E}}(X) \cap T_{\mathrm{R}}(X), \\
T_{\mathrm{SER}}(X) & =T_{\mathrm{SE}}(X) \cap T_{\mathrm{R}}(X), \\
T_{\mathrm{EO}}(X) & =T_{\mathrm{E}}(X) \cap T_{\mathrm{O}}(X), \\
T_{\mathrm{SEO}}(X) & =T_{\mathrm{SE}}(X) \cap T_{\mathrm{O}}(X), \\
T_{\mathrm{OR}}(X) & =T_{\mathrm{O}}(X) \cap T_{\mathrm{R}}(X) .
\end{aligned}
$$

It is known that the intersection of subsemigroups of a semigroup $S$ is either an empty set or itself a subsemigroup of $S$. Then $T_{\mathrm{ER}}(X), T_{\mathrm{SER}}(X)$,
$T_{\mathrm{EO}}(X), T_{\mathrm{SEO}}(X)$ and $T_{\mathrm{OR}}(X)$ are subsemigroups of $T(X)$ containing $i_{X}$.

In this paper the set $X$ under consideration is a totally ordered set with $E$ an arbitrary equivalence relation on $X$. We denote by $X / E$ the family of $E$ classes on $X$ and by $|A|$ the cardinality of a set $A$.

\section{MAIN RESULTS}

In this section, we characterize the conditions under which some of above subsemigroups of $T(X)$ are equal.

Theorem $1 T_{\mathrm{ER}}(X)=T_{\mathrm{R}}(X)$ if and only if for all $A, B \in X / E$ such that $A \neq B$, if there exist $a \in A$, $b \in B$ such that $a<b$, then $|B|=1$.

Proof: Assume that there exist $A, B \in X / E$ such that $A \neq B$, if $a<b$ for some $a \in A, b \in B$ and $|B|>1$. Define $\alpha: X \rightarrow X$ by

$$
x \alpha= \begin{cases}a, & \text { if } x=b, \\ x, & \text { otherwise }\end{cases}
$$

Clearly, $x \alpha \leqslant x$ for all $x \in X$ and then $\alpha \in$ $T_{\mathrm{R}}(X)$. Let $c \in B \backslash\{b\}$. Thus $(b, c) \in E$. Since $(b \alpha, c \alpha)=(a, c) \notin E, \alpha \notin T_{\mathrm{E}}(X)$. Therefore $T_{\mathrm{ER}}(X) \neq T_{\mathrm{R}}(X)$.

Conversely, suppose that for all $A, B \in X / E$ such that $A \neq B$, if there exist $a \in A, b \in B$ such that $a<b$, then $|B|=1$. To show that $T_{\mathrm{ER}}(X)=$ $T_{\mathrm{R}}(X)$, let $\alpha \in T_{\mathrm{R}}(X)$ and let $x, y \in X$ be such that $(x, y) \in E$. Hence $x, y \in B$ for some $B \in X / E$. If $x=y$, then $(x \alpha, y \alpha) \in E$. Suppose that $x \neq y$. Then $|B|>1$. Since $\alpha \in T_{\mathrm{R}}(X), x \alpha \leqslant x$. It follows 
by assumption that $x \alpha \in B$. Similarly, we have that $y \alpha \in B$. This means that $(x \alpha, y \alpha) \in E$. Therefore, $\alpha \in T_{\mathrm{E}}(X)$ and hence $T_{\mathrm{ER}}(X)=T_{\mathrm{R}}(X)$.

Theorem $2 T_{\mathrm{ER}}(X)=T_{\mathrm{E}}(X)$ if and only if $|X|=$ 1.

Proof: Suppose that $|X|>1$. Let $a, b \in X$ be such that $a \neq b$. Then there exist $A, B \in X / E$ such that $a \in A$ and $b \in B$. Suppose that $a<b$ and define $\alpha: X \rightarrow X$ by

$$
x \alpha= \begin{cases}b, & \text { if } x \in A, \\ x, & \text { otherwise }\end{cases}
$$

Let $x, y \in X$ be such that $(x, y) \in E$. Then

$$
(x \alpha, y \alpha)= \begin{cases}(b, b) \in E, & \text { if } x, y \in A, \\ (x, y) \in E, & \text { otherwise }\end{cases}
$$

which implies that $\alpha \in T_{\mathrm{E}}(X)$. Since $a \alpha=b \nless a$, we deduce that $\alpha \notin T_{\mathrm{ER}}(X)$.

Theorem $3 T_{\mathrm{SER}}(X)=T_{\mathrm{R}}(X)$ if and only if $E=$ $X \times X$.

Proof: Suppose that $E \neq X \times X$. Then there exist $a, b \in X$ such that $(a, b) \notin E$. Suppose that $a<b$ and define $\alpha: X \rightarrow X$ as given in Theorem 1. Then $\alpha \in$ $T_{\mathrm{R}}(X)$. Since $(b, b \alpha)=(b, a) \notin E, \alpha \notin T_{\mathrm{SE}}(X)$. Hence $T_{\mathrm{SER}}(X) \neq T_{\mathrm{R}}(X)$.

Assume that $E=X \times X$. We have that $T_{\mathrm{SE}}(X)=T(X)$, hence $T_{\mathrm{SER}}(X)=T_{\mathrm{SE}}(X) \cap$ $T_{\mathrm{R}}(X)=T(X) \cap T_{\mathrm{R}}(X)=T_{\mathrm{R}}(X)$.

Theorem $4 T_{\mathrm{SER}}(X)=T_{\mathrm{SE}}(X)$ if and only if $E=$ $I_{X}$ where $I_{X}$ is the identity relation on $X$.

Proof: Suppose that $E \neq I_{X}$. Then there exist $a, b \in$ $X$ such that $a \neq b$ and $(a, b) \in E$. We may assume that $a<b$ and define $\alpha: X \rightarrow X$ by

$$
x \alpha= \begin{cases}b, & \text { if } x=a, \\ x, & \text { otherwise }\end{cases}
$$

For each $x \in X$,

$$
(x, x \alpha)= \begin{cases}(a, b) \in E, & \text { if } x=a, \\ (x, x) \in E, & \text { otherwise }\end{cases}
$$

hence $\alpha \in T_{\mathrm{SE}}(X)$. Since $a \alpha=b \not a$, we conclude that $\alpha \notin T_{\mathrm{R}}(X)$. Therefore $T_{\mathrm{SER}}(X) \neq T_{\mathrm{SE}}(X)$.

Conversely, suppose that $E$ is the identity relation on $X$. Let $\alpha \in T_{\mathrm{SE}}(X)$ and $x \in X$. Then we have that $(x, x \alpha) \in E$. By assumption, $x \alpha=x$ which implies that $\alpha \in T_{\mathrm{R}}(X)$. This proves that $T_{\mathrm{SE}}(X) \subseteq T_{\mathrm{R}}(X)$, hence $T_{\mathrm{SE}}(X)=T_{\mathrm{SE}}(X) \cap$ $T_{\mathrm{R}}(X)=T_{\mathrm{SER}}(X)$.
Theorem $5 T_{\mathrm{EO}}(X)=T_{\mathrm{O}}(X)$ if and only if $E=$ $X \times X$ or $E=I_{X}$.

Proof: Assume that $E \neq X \times X$ and $E \neq I_{X}$. Then there exist $A, B \in X / E$ such that $|A|>1$ and $B \neq$ $A$. Let $a, c \in A$ be such that $a<c$ and $b \in B$. Since $E$ is an equivalence relation on $X$, we have that $b \in B \backslash A$. Define $\alpha: X \rightarrow X$ by

$$
x \alpha= \begin{cases}\max (b, c), & \text { if } c \leqslant x, \\ \min (b, c), & \text { otherwise. }\end{cases}
$$

To show that $\alpha \in T_{\mathrm{O}}(X)$, let $x, y \in X$ be such that $x \leqslant y$.

Case 1. If $c \leqslant x \leqslant y$ or $x \leqslant y<c$, then we get that $x \alpha=y \alpha$.

Case 2. If $x<c \leqslant y$, then we have that $x \alpha=$ $\min (b, c)<\max (b, c)=y \alpha$.

From two cases, we deduce that $\alpha \in T_{\mathrm{O}}(X)$. Since $(a, c) \in E$ and $(a \alpha, c \alpha)=(\min (b, c), \max (b, c)) \notin$ $E$, we deduce that $\alpha \notin T_{\mathrm{E}}(X)$. This proves that $T_{\mathrm{EO}}(X) \neq T_{\mathrm{O}}(X)$ as required.

Conversely, suppose that $E=X \times X$ or $E=$ $I_{X}$. We then have that $T_{\mathrm{E}}(X)=T(X)$. Therefore $T_{\mathrm{EO}}(X)=T_{\mathrm{E}}(X) \cap T_{\mathrm{O}}(X)=T(X) \cap T_{\mathrm{O}}(X)=$ $T_{\mathrm{O}}(X)$.

Theorem $6 T_{\mathrm{EO}}(X)=T_{\mathrm{E}}(X)$ if and only if $|X|=$ 1.

Proof: Assume that $|X|>1$. Let $a, b \in X$ be such that $a<b$. Then $a \in A$ for some $A \in X / E$. Define $\alpha: X \rightarrow X$ by

$$
x \alpha= \begin{cases}b, & \text { if } x \in A \text { and } x \neq b, \\ a, & \text { otherwise. }\end{cases}
$$

To show that $\alpha \in T_{\mathrm{E}}(X)$, let $x, y \in X$ be such that $(x, y) \in E$. Then $x, y \in B$ for some $B \in X / E$.

Case 1. $B \neq A$. This implies that $x, y \notin A$. Hence $(x \alpha, y \alpha)=(a, a) \in E$.

Case 2. $B=A$. If $b \in A$, then $x \alpha, y \alpha \in$ $\{a, b\} \subseteq A$. Thus $(x \alpha, y \alpha) \in E$. If $b \notin A$, then $x \neq b$ and $y \neq b$ which implies that $(x \alpha, y \alpha)=(b, b) \in E$. From two cases, we then have $\alpha \in T_{\mathrm{E}}(X)$. Since $a<b$ and $a \alpha=b \notin a=b \alpha$, we get $\alpha \notin T_{\mathrm{O}}(X)$. Hence $T_{\mathrm{EO}}(X) \neq T_{\mathrm{E}}(X)$.

Theorem $7 T_{\mathrm{SEO}}(X)=T_{\mathrm{SE}}(X)$ if and only if $E=$ $I_{X}$.

Proof: Assume that $E \neq I_{X}$. Then there exist $a, b \in$ $X$ such that $(a, b) \in E$ and $a \neq b$. We may assume 
that $a<b$ and define $\alpha: X \rightarrow X$ by

$$
x \alpha= \begin{cases}b, & \text { if } x=a, \\ a, & \text { if } x=b, \\ x, & \text { otherwise }\end{cases}
$$

It is easy to verity that $\alpha \in T_{\mathrm{SE}}(X)$. Since $a<b$ and $a \alpha>b \alpha, \alpha \notin T_{\mathrm{O}}(X)$. Therefore $T_{\mathrm{SEO}}(X) \neq$ $T_{\mathrm{SE}}(X)$.

Conversely, suppose that $E=I_{X}$. Then we get $T_{\mathrm{SE}}(X)=\left\{i_{X}\right\}$. It follows that $T_{\mathrm{SEO}}(X)=$ $T_{\mathrm{SE}}(X) \cap T_{\mathrm{O}}(X)=\left\{i_{X}\right\} \cap T_{\mathrm{O}}(X)=\left\{i_{X}\right\}=$ $T_{\mathrm{SE}}(X)$.

Theorem $8 T_{\mathrm{SEO}}(X)=T_{\mathrm{O}}(X)$ if and only if $E=$ $X \times X$.

Proof: Suppose that $E \neq X \times X$. Then there exist $a, b \in X$ such that $(a, b) \notin E$. We may assume that $a<b$ and define $\alpha: X \rightarrow X$ by

$$
x \alpha= \begin{cases}b, & \text { if } x \geqslant a, \\ a, & \text { otherwise }\end{cases}
$$

To show that $\alpha \in T_{\mathrm{O}}(X)$, let $x, y \in X$ be such that $x \leqslant y$.

Case 1. If $a \leqslant x \leqslant y$ or $x \leqslant y<a$, then we get $x \alpha=y \alpha$.

Case 2. If $x<a \leqslant y$, then we have that $x \alpha=$ $a<b=y \alpha$.

From two cases, we deduce that $\alpha \in T_{\mathrm{O}}(X)$. Since $(a, a \alpha)=(a, b) \notin E, \alpha \notin T_{\mathrm{SE}}(X)$. Hence $T_{\mathrm{SEO}}(X) \neq T_{\mathrm{O}}(X)$.

Conversely, assume that $E=X \times X$. Thus $T_{\mathrm{SE}}(X)=T(X)$. Hence $T_{\mathrm{SEO}}(X)=T_{\mathrm{SE}}(X) \cap$ $T_{\mathrm{O}}(X)=T(X) \cap T_{\mathrm{O}}(X)=T_{\mathrm{O}}(X)$.

Theorem $9 T_{\mathrm{OR}}(X)=T_{\mathrm{O}}(X)$ if and only if $|X|=$ 1.

Proof: Assume that $|X|>1$. Then there exist $a, b \in$ $X$ such that $a<b$. Define $\alpha: X \rightarrow X$ as given in Theorem 8. Then $\alpha \in T_{\mathrm{O}}(X)$. Since $a \alpha=b>$ $a, \alpha \notin T_{\mathrm{R}}(X)$. Hence $T_{\mathrm{OR}}(X) \neq T_{\mathrm{O}}(X)$.

Theorem $10 T_{\mathrm{OR}}(X)=T_{\mathrm{R}}(X)$ if and only if $|X| \leqslant$ 2.

Proof: Suppose that $|X|>2$. Let $a, b, c \in X$ be such that $a<b<c$. We define $\alpha: X \rightarrow X$ by

$$
x \alpha= \begin{cases}a, & \text { if } x=c, \\ x, & \text { otherwise }\end{cases}
$$

Then $\alpha \in T_{\mathrm{R}}(X)$. We note that $b<c$ and $b \alpha=b \not$ $a=c \alpha$. Thus $\alpha \notin T_{\mathrm{O}}(X)$ and so $T_{\mathrm{OR}}(X) \neq T_{\mathrm{R}}(X)$.

Conversely, assume that $|X| \leqslant 2$. Let $\alpha \in$ $T_{\mathrm{R}}(X)$. To show that $\alpha \in T_{\mathrm{OR}}(X)$, let $x, y \in X$ be such that $x<y$. Since $\alpha \in T_{\mathrm{R}}(X)$ and by assumption, we then have $x \alpha=x$ (since $x \alpha \leqslant x$ ). It follows that $x \alpha \leqslant y \alpha$ (since $y \alpha=x$ or $y \alpha=y$ ), which implies that $\alpha \in T_{\mathrm{O}}(X)$. Therefore $T_{\mathrm{OR}}(X)=$ $T_{\mathrm{R}}(X)$.

\section{REFERENCES}

1. Higgins $P$ (1993) Combinatorial results for semigroups of order-preserving mappings. Math Proc Camb Phil Soc 113, 281-96.

2. Namnak C, Laysirikul E (2012) A note on the regularity for semigroups of self- $E$-preserving transformations. Proceedings of the 1st AGRC 2012, ST164-167.

3. Pei H (2005) Regularity and Green's relations for semigroups of transformations that preserve an equivalence. Comm Algebra 33, 109-18. 